(Cancelliere \& Kertesz, 1990). I would argue that it is highly likely that the AC influences both the verbal and prosodic aspects of aggressive vocalisations, possibly by separate mechanisms. This issue certainly deserves further study.

A second contention relates to the strength of the evidence for a lack of emotional content in the vocalisations elicited from the cingulum. Careful reading of sources for my article will reveal the original study by Jurgens et al (1967) which supports this strand of evidence. This was a direct electrical stimulation experiment in 13 squirrel monkeys in which it was found that vocalisations elicited from, among other brain structures, the cingulum were identified as (neutral)contact and food calls, whereas those elicited from the amygdala group had an aggressive function.

The authors imply that the role of dopamine should be questioned because dopamine antagonists are effective in "only" $60-70 \%$ of cases. This is strikingly effective by psychiatric standards. One need only refer to the variable response of schizophrenic patients to dopamine antagonists to realise that the case for dopamine in TS is, if anything, stronger on this basis. I fully accept, as was discussed in the original article, that 5-hydroxytryptamine (5-HT) may well be directly or indirectly implicated in TS. The fact that 5-HT reuptake inhibitors produce such an unpredictable response in TS, however, argues for a more peripheral role of 5-HT.

Handley et al contend that the fact "that there is an input of dopamine to the amygdala is not an argument for (its) involvement (in TS) since such inputs exist to many other parts of the brain". This is a logical error. Involvement of a neurotransmitter in a disease process does not preclude that neurotransmitters' role in other parts of the brain. Their argument would negate the role of dopamine in the aetiology of schizophrenia or Parkinson's disease. It is indeed one of the advances of modern psychopharmacology that the involvement of various neurotransmitters in disease processes is conceptualised as a problem of imbalance rather than absolute excess or deficit of a particular neurotransmitter system in the brain.

The role of the cingulum in vocalisations is reasonably well established. As outlined in the original article, my hypothesis does not exclude the role of other limbic and subcortical structures in the pathophysiology of TS. Rather, the proposed model highlights the key role of the AC in the disorder, particularly with reference to the neglected aspect of the aggressive content of the vocalisation of TS.

Cancellaere, A. E. B. \& Kertesz, A. (1990) Lesion localisation in acquired deficits of emotional expression and comprehension. Brain Cognition, 13, 133-147.
Jugens, U., Maurus, M., Ploog, D., et al (1967) Vocalisation in the squirrel monkey (Saimiri sciureus) elicited by brain stimulation. Experimental Brain Research, 4, 114-117.

School of Psychiatry and Behavioural Sciences

DANITZa JADRESIC

Rawnsley Building

Manchester Royal Infirmary

Oxford Road

Manchester M139WL

\section{Tattooed female psychiatric patients}

SIR: Inch \& Huws (Journal, January 1993, 162, 128-129) concluded that in their four cases of female psychiatric patients, tattoos were a stigma of sexual abuse because all four women gave a history of sexual abuse in childhood. However, they did not comment on the fact that all four patients had borderline personality disorder, and at least two were also dependent on alcohol (the status of alcohol dependence for the other two was not declared).

There are numerous theories trying to explain the association of tattoos with a specific psychiatric diagnosis. Ferguson-Rayport et al (1955) suggested that the tattoo's content has diagnostic significance. However, failing to find any correlation between psychiatric diagnosis and tattoo theme or content, Gittleson et al (1969) suggested that although its artistic content does not correlate with specific diagnosis, the mere presence of a tattoo does. Patients with a Cluster B personality disorder (DSM-III - R) (which includes antisocial and borderline personality disorders, among others) have a history of divorce, chaotic lifestyle, aggressive outbursts, impulsivity, and lack of control. The strongest association between tattoos and diagnosis is found in patients suffering from these disorders (Raspa \& Cusack, 1990). Second to personality disorder, alcohol and substance abuse show strongest association with tattoos (Buhrich, 1983).

It is thought that an important function of tattoos is that they denote a sense of belonging and affiliation to an idealised group. In primitive cultures, tattoos identified a member of a particular tribe. In the West, tattoos are observed in people belonging to particular segments of society (e.g. sailors, criminals, addicts, etc.). Tattoos have been characterised as an artificial embellishment of the body boundary - a prosthetic attempt to strengthen one's sense of ego definition. Patients with borderline personality disorder have identity disturbance (DSM-III - R) and have an unstable sense of self. A need to affiliate with a group to achieve some amount of stability and identity may be fulfilled by tattooing (thus being 
recognised as a part of a group or culture). These patients also show sudden shifts from overidealisation to devaluation, which may be the reason for adopting the tattoos of an idealised culture at first, but making serious efforts to get rid of them subsequently.

An association of tattoos with borderline personality disorder is now recognised, and Inch \& Huws' four cases further substantiate that association. The conclusion (from their short note) that tattoos may be a stigma of sexual abuse in childhood should not be entertained at this stage.

BUFrich, N. (1983) The association of erotic piercing with homosexuality, sadomasochism, bondage, fetishism, and tattoos. Archives of Sexual Behaviour, 12, 167-171.

Ferguson-Rayport, S., Grifftrh, R. \& Straus, E. (1955) The psychiatric significance of tattoos. Psychiatry Quarterly, 29, 112-131.
Gittlason, N. L., Wallen, G. D. \& Dawson-Butterwoth, K. (1969) The tattooed psychiatric patient. British Journal of Psychiatry, 115, 1249-1253.

RAspA, R. F. \& CusAck, J. (1990) Psychiatric implications of tattoos. American Family Physician, 41, 1481-1486.

Department of Psychiatry

Dinesh K. ARYA 'A' Floor, South Block

Queen's Medical Centre

Nottingham NG7 2UH

\section{CORRIGENDUM}

Journal, March 1993, 162, 426. The parentheses in the second column, at the end of the second paragraph, should read "(life-threatening 5-HT syndrome of hyperthermia)".

\section{A HUNDRED YEARS AGO}

\section{The legal fiction of the insanity of suicides}

The verdict returned by the jury as the result of the inquest on Mr Lombard John Newman Tanner, LRCP, SEd, who was found dead in his bedroom at a hotel in Fleet Street on April 29th, opens up once more the question of the justifiability of verdicts of "Suicide during temporary insanity" in the absence of any evidence of insanity. Both jurymen and judges are as eager to admit a plea of insanity when a man makes away with himself as they are unwilling to do so when he takes the life of another. The legal fiction - for in many cases it is nothing else - is now a mere "formula" which has outlived the purpose it was meant to serve. A felo de se is no longer buried at crossroads with a stake through his heart, nor does suicide entail any consequences on the survivors of a kind rendering any legal subterfuge desirable in their interests. Is not the supposed charity to the dead man in assuming that he was insane because he destroyed himself much more than counterbalanced by the injustice done to his relatives by branding the family, with an undeserved social stigma? Morselli, in his great work Suicidio, says that in only about one-third of all cases of suicide are the perpetrators of the rash act insane; and Dr Wynn Westcott states that in only 20 per cent of the cases of suicide which have come before him as coroner had the deceased shown signs of mental unsoundness. In the case of Mr Tanner, the facts showed clearly that the suicide was deliberate, and there was not the slightest evidence of insanity. Dr Tanner, MP (with whom all will sympathise in the affliction that has befallen him), stated at the inquest that his brother took his own life "calmly, conscientiously, and according to his lights;" and charity as well as justice may fairly be satisfied with this pronouncement. It would be going outside our province to discuss the ethical aspects of self-murder; all that we are concerned with here is to urge, as we have done before, that for a jury to return a verdict imputing insanity to a man without any evidence beyond the bare fact of suicide is an unworthy evasion.

\section{Reference}

Lancet, 6 May 1893, 972. 